\title{
Mast cell activation syndrome (MCAS) diagnosed using double-balloon enteroscopy
}

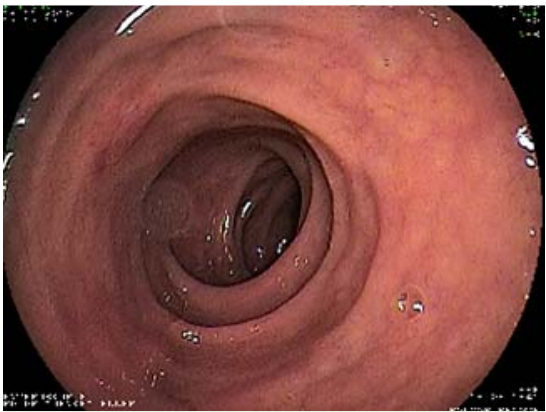

Fig. 1 Jejunal mucosa with mild edema.

A 69-year-old woman was referred for small-bowel endoscopy because of abdominal pain and diarrhea of 1-year's duration. She complained of bloating and fullness of her abdomen, accompanied by headaches, occasional palpitations, and anxiety. Her past medical history was positive for coronary artery disease, hypertension, and hyperlipidemia. An outpatient colonoscopy revealed diverticula.

Esophagogastroduodenoscopy, including biopsies of the stomach and duodenum, was normal. Clinical examination was unremarkable except for dermographism. Laboratory data were within reference ranges. Special laboratory tests including vanillylmandelic acid, epinephrine, calcitonin, and chromogranin A were normal. The patient underwent an oral doubleballoon enteroscopy (DBE), which disclosed non-specific findings such as edema and minimal erythema of the jejunal mucosa ( Fig. 1). Histopathological analysis revealed mast cell infiltration of the jejunal mucosa ( $\bullet$ Fig. 2). A bone marrow aspirate and a skin biopsy were negative. A diagnosis of mast cell activation syndrome (MCAS) was made, and the patient was placed on sodium chromoglycate, ranitidine, and $\mathrm{H} 1$ blockers. Her symptoms gradually improved and at 10 -months follow-up she remained clinically stable. Mast cell activation disease (MCAD) comprises a collection of disorders characterized by accumulation of genetically altered mast cells which, potentially, can

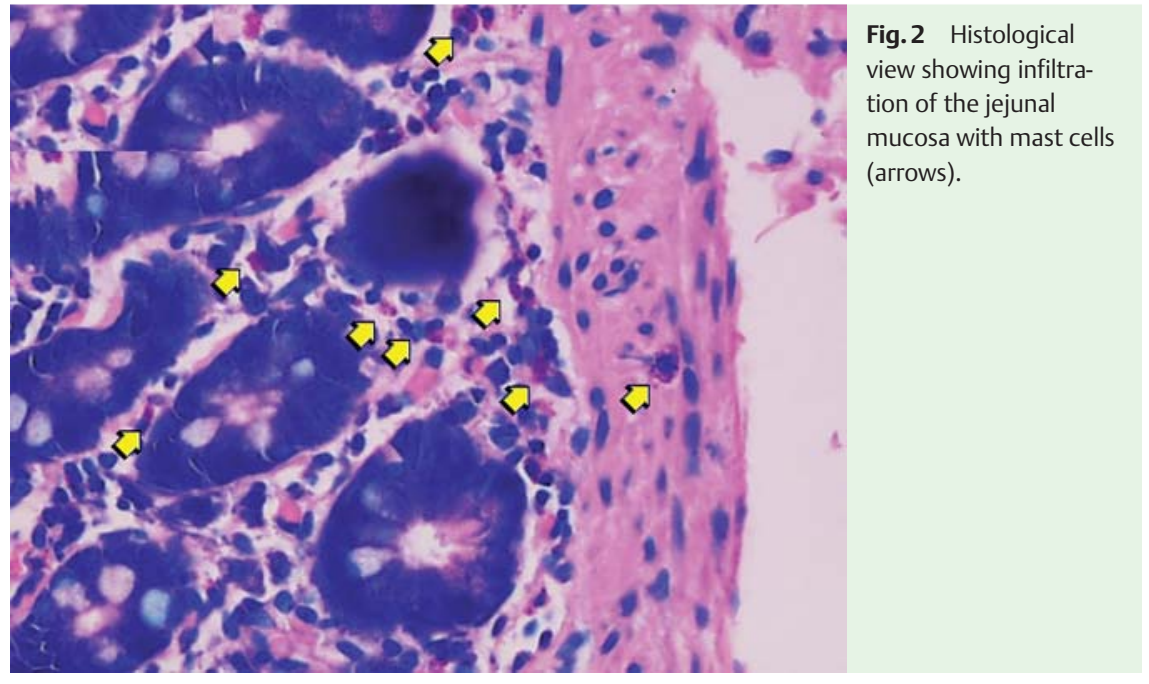

affect the function of any organ [1]. MCAD is now classified into indolent mastocytosis, isolated bone marrow mastocytosis, smoldering systemic mastocytosis, systemic mastocytosis with clonal hematologic disorder, aggressive systemic mastocytosis, and mast cell leukemia [1]. In contrast, patients with MCAS have the typical symptoms of MCAD but fail to meet the classic World Health Organization (WHO) criteria for systemic mastocytosis [1,2]. Furthermore, MCAS often does not result in any abnormalities identified during laboratory testing, including tryptase and chromogranin A levels [1-3]. Nevertheless, patients with MCAS suffer from the same symptoms as patients with systemic mastocytosis [1-3]. Thus, MCAS may be an important and probably unrecognized cause of irritable bowel syndrome (IBS) [1,3].

Any endoscopist dealing with patients with IBS-like symptoms should therefore include MCAS as a possible cause of the patient's symptoms and look for this condition. Because mast cells tend to be destroyed during activation, it is not always feasible to find them during routine biopsies [3]. In addition, systemic gastrointestinal inflammatory disorders such as Crohn's disease and celiac disease are also known to affect the small bowel in a patchy pattern. Thus obtaining multiple biopsies of even normal-appearing mucosa from various sites in the gastrointestinal tract and communicating with the pathologist to specifically search for mast cells may improve the recognition of this condition.

To the best of our knowledge, this is the first report on the utility of DBE for the diagnosis of MCAS. This report should serve as an impetus to consider MCAS or MCAD in patients with IBS-like symptoms presenting for small-bowel endoscopy.

\section{Endoscopy_UCTN_Code_CCL_1AC_2AH}

\section{Competing interests: None}

\section{K. Mönkemüller ${ }^{1,2}$, M. Kassalik' ${ }^{1}$, D. Baraksei ${ }^{1}$, L. C. Fry ${ }^{1}$, J. Moege ${ }^{3}$}

${ }^{1}$ Division of Gastroenterology, Hepatology and Infectious Diseases, Otto-von-Guericke University, Magdeburg, Germany

2 Department of Internal Medicine, Gastroenterology and Infectious Diseases, Marienhospital Bottrop, Bottrop, Germany

${ }^{3}$ Praxis Moege, Bottrop, Germany 


\section{References}

1 Molderings GJ, Brettner S, Homann J et al. Mast cell activation disease: a concise practical guide for diagnostic workup and therapeutic options. J Hematol Oncol 2011; 4: $10-18$

2 Akin C, Valent P, Metcalfe DD. Mast cell activation syndrome: proposed diagnostic criteria. J Allergy Clin Immunol 2010; 126 : 1099-1104

3 Hamilton MJ, Hornick JL, Akin C et al. Mast cell activation syndrome: a newly recognized disorder with systemic clinical manifestations. J Allergy Clin Immunol 2011; 128: $147-152$
Bibliography

DOI http://dx.doi.org/

10.1055/s-0031-1291587

Endoscopy 2012; 44: E72-E73

(C) Georg Thieme Verlag KC

Stuttgart · New York

ISSN 0013-726X
Corresponding author

K. Mönkemüller, MD, PhD, FASGE

Division of Gastroenterology,

Hepatology and Infectious Diseases

Marienhospital Bottrop

Josef-Albers-Straße 70

46242 Bottrop

Germany

Fax: + 49-2041-1016-1019

moenkemueller@yahoo.com 